\title{
11
}

\section{Early Recognition Method: ‘Opening Doors' in Risk Management Dialogue Between Mental Health and Prison Services}

Frans Fluttert, Gunnar Eidhammer, and Karl Yngvar Dale

\section{Introduction}

Working in a closed secure facility requires a lot of employees to keep the work environment safe. Various studies show that prison employees are confronted with a high degree of stress, which can lead to burnout (Andersen et al., 2017; Bezerra \& De Mahalhães, 2016; Finney et al., 2013; Gadon et al., 2006). The most important risk factors in burnout

The content is based on the article:

Fluttert, F.A.J. \& G. Eidhammer (2018). ERM-Vroegsignalering opent de deur naar risicomanagement observaties en de dialoog met gedetineerden. Proces, 97-1, 25-39.

G. Eidhammer

Oslo University Hospital, Oslo, Norway

F. Fluttert $(\varangle) \cdot$ K. Y. Dale

Molde University College (MUC), Molde, Norway

e-mail: f.fluttert@pcvanmesdag.nl

K. Y. Dale

e-mail: karl.y.dale@himolde.no 
are high work pressure, interaction problems with colleagues, supervisors or prisoners, the emotional burden of work and organisational problems. The work pressure is seriously increased in cases of violence against employees; a constant feeling of 'a malaise is in the air' is stressful (Bezerra \& De Mahalhães 2016). Despite this observation, Andersen et al. (2017) found no direct relation between burnout among prison employees and violence of prisoners in a study of more than 3000 penitentiary workers. Research in other secure institutions, however, has shown violence from clients towards employees to have a major impact, eliciting feelings of anger, fear and gloom (Finney et al., 2013; van Leeuwen and Harte, 2016).

In order to explain this violence, the emphasis is often on the personal characteristics of clients. However, it appears that situational, relational and environmental factors also make an important contribution to the explanation of the origin of violence (Bjørkly et al., 2019). For example, overcrowding and insufficient limiting of undesirable behaviour are associated with an increased risk of violence. Decisions about the risk of violence tend to be based on studies with a large number of subjects (clients) (Bezerra \& De Mahalhães, 2016; Carlsson et al., 2006; Newbill, 2010; Nijman et al., 1997). But it is as important in everyday practice that violence be understood and influenced at an individual level through exploring the behaviour of the potentially violent patient as an individual.

In secured institutions in psychiatry, such as Forensic Psychiatric Clinics [FPC], risk management strategies are well developed and applied to manage and control violence in individual patients. The two most applied risk management models explaining the vision behind risk management strategies are: (1) the Good Lives Model [GLM] and (2) the Risk-Needs-Responsivity [RNR] model. The GLM explains that the focus of risk management should be on assisting the client to develop meaningful life plans for rehabilitation, whereas in the RNR model it is argued that the focus in treatment should be on a structured professional judgement of criminogenic factors matching the patient's risk profile, the needs for treatment and the patient's abilities to comply (Andrew, 2012; Ward, 2002). In the literature and research addressing risk management approaches, the debate about the efficacy of GLM compared to RNR 
continues (Looman \& Abracen, 2013). Some researchers argue that, apart from the focus of risk management, there is much overlap between the models both addressing patient's social factors that contribute to a 'good life' without offending. Most research studying the effectiveness of risk management has focused on the RNR model, showing it to contribute significantly to relapse prevention and reduced reoffending (Looman \& Abracen, 2013; Taxman et al., 2013). It is argued that the RNR model facilitates the patients' interaction with care providers, i.e. the professionals learn, in a structured way, to understand how violence can occur, what the consequences may be and especially, how they can control aggressive feelings and behaviours in patients (Douglas et al., 2013). The ERM Early Recognition Method (ERM; Fluttert et al., 2008) is a risk management strategy fitting, in its origins, within the RNR model. The uniqueness of ERM lies, however, as a risk management strategy that emphasises the importance of having the patient involved in the risk assessment, identifying and managing, in structured way, specifically the early warning signs of violence. The ERM strategy focuses on describing early warning signs of behavioural escalation in ERM-plans. The focus is not on unravelling and describing the crisis but on avoiding it and the development of behavioural stability by means of managing early warning signs. ERM research shows that its application in the FPC context contributes to less frequent and less serious violence (Fluttert et al., 2010b).

In the prison context, prisoners also benefit from (treatment) programmes that can provide insight into their behavioural problems and how they can manage these. Here too, the RNR principle is an important starting point. That this is an internationally recognised principle is evident from the fact that the Handbook on the Management of highrisk prisoners UNODC (United Nations Office on Drugs and Crime, 2016), advocates the development and application of risk management programmes according to the RNR principles. The importance of this is also illustrated by Campbell et al. (2010) in a study of 22 prisoners: that showed that offender treatment programmes' using RNR principles contributed to reduced recidivism among prisoners. These prisoners reported that they wanted to improve their lives but did not know how to achieve this. Prison programmes can help with this. These programmes 
are mostly aimed at the prisoners' return to society, where their problems become manageable and social integration is promoted. Lesser attention is paid to the application of risk management strategies that prison employees can apply in the day-to-day management of prisoners when they are in the prison itself. The ERM has the potential to address this offering a tool with which to identifying and discussing with prisoners the early warning signs of violence.

Inpatient violence in forensic institutions and violence in prisons evoke fear and stress among employers working in these facilities (Finney et al., 2013; Leeuwen \& Harte, 2016). The nature of violence from prisoners towards staff is comparable to the violence from forensic patients towards staff. In both contexts staff are confronted with verbally and physically threatening behaviours (Andersen et al., 2017; van Leeuwen et al., 2016). Despite this, there are hardly any risk management strategies assisting care-givers or prison staff within the prison to understand and manage violence, and reduce violent incidents before they begin. Moreover, there are hardly any risk management strategies in which the prisoner is actively involved in this risk management (Eidhammer et al., 2014; Ray \& Simpson, 2019).

\section{Aims}

The aims of this chapter are to first explore the complexity and multifactorial nature of violence and its development. It then explains why a structured strategy based on a risk management model is necessary to adequately assess and manage violence. Finally, it addresses how the knowledge and research of the ERM-application in forensic psychiatry services could be transferred and be successfully applied as a violence reduction strategy in prison services. We also explore how risk assessment is an interagency issue and how the ERM can form a means of establishing a dialogue between services and actors. We view this aim as a form of social innovation: 'the development and implementation of new ideas (products, services and models) to meet social needs .... They are innovations that are not only good for society but enhance individuals' capacity to act' (European Commission, 2013, p. 6). These can take 
the form of new ways of working altogether but can take also the form of the transfer of effective models from one discipline to another. The transfer of the ERM model of risk management into the prison environment represents, therefore, a form of service social innovation. In order to explain this bridge being constructed between risk management between forensic- and prison services, we draw on studies in Norway and the Netherlands where the ERM has contributed to the better management of early warning signs of behavioural escalation and prevention of violence (Eidhammer et al., 2014).

\section{Multifactorial nature of violence and its development}

In order to comprehend the complexity of violence, multifactor models explain which factors influence the process towards aggression. We address three angles, from which to understand clients getting aggressive or violent. First, we will explain, by means of the General Aggression Model, how knowledge structures are related to the development of aggression. Next, we will reflect on how criminal attitudes and thinking styles could be understood and effected in treatment. Finally, we will, by means of the Hiday model (Hiday, 1997, 2006), connect the broad spectrum of influential factors and possible violence.

The General Aggression Model (GAM) is framework explaining two main aspects of aggression: the individual's present state, and the individual episodes of aggression. The development and occurrence of aggression is influenced by knowledge structures such as beliefs, perceptual schemata (e.g. perceiving events as hostile), expectation schemata and behavioural scripts (e.g. problems have to be solved with aggression). These knowledge structures affect the following social-cognitive phenomena: the perception of a situation, the interpretation of what is happening, the decision on how to act and the resulting behaviours, e.g. aggression. The knowledge structures are the results of an individual's experiences and perception of social events. Hence, each episode of aggression could serve as a learning trail through which repetitive aggression can be predicted. 
A second perspective on violence is offered by Bulten et al. (2009) who conducted research into dimensions in criminal thinking styles among prisoners. Three dimensions of thinking styles can be distinguished, namely: (1) pro-active, anti-social and narcissistic, (2) emotional, reactive and impulsive and, (3) positive, open-minded and pro-social. These thinking styles are not per se separately inhibited in individuals, but each of these are mostly present in a dominant way. Bulten et al. suggested that prisoners with emotional, reactive and impulsive thinking styles could benefit especially from interventions aimed at learning to recognise thoughts that cause them problems.

Compared to the General Aggression Model and Bulten's study, Hiday (2006) developed a more comprehensive model emphasising primarily the main factors related to violence and how they are connected. These factors can interact and influence each other, leading to violence. The Hiday model highlights the importance of identifying personal and contextual factors proposing that there is no single pathway between a mental disorder and violence. Rather, there are many factors that mediate the relationship. For example, the mental illness in psychotic clients (who exhibit violence) cannot be the 'only' explanatory factor. It is also brought on by tense situations, suspiciousness, use of alcohol/drugs and so on.

The models explained above strengthen our understanding of why the identification of early warning signs of aggression and violence are valuable for risk management. For example, in the General Aggression Model and illustrated by Bulten et al. (2009) it is illustrated that the person's cognitive processes such as affect, arousal and thinking style benefit from a systematic approach, an approach that guides the 'decision process' at play when the individual decides whether to engage in aggression behaviours and how they do this. Hiday (2006) added to understanding of this process through explaining the interaction between violence inducing factors and how violence develops from these. Drawing on these principles the core idea of the ERM is that clients in psychiatric institutions, and probably also prisoners, within this process can learn to recognise and manage specifically their early warning signs of violence in order to avoid escalation. By means of this ERM client's awareness of their pathway into violence, they are able to appraise and articulate their own thoughts \& behaviours through reflecting on their 
early warning signs as drawn up in the ERM tool. The aim of the tool is for the users to describe client-specific warning signs, an imminent violent episode, preferably in relation to the client himself. Important in this process is that the client is involved in order to get a 'customised plan' (Fluttert et al., 2008). Prisoners with more pro-active, anti-social and narcissistic thinking styles will hardly profit from strategies aimed at gaining insight in their behaviours because they do not perceive themselves as a problem. In cases of psychopaths these strategies should be avoided, unequivocally, because insight could serve as 'ammunition' to manipulate others, even more.

Both for the symptoms of a disorder and for its expression, there are almost always early warning signs (Fluttert et al., 2008). Tension and risk behaviours accumulate over time and are almost always preceded by the same early warning signs, called 'signature risk'signs or 'the signature of the behaviour' (Birchwood, 2000; Fluttert et al., 2008). These are signs that are very personal to the individual and often 'repetitive' when repeated stress build up occurs. The problem is that such warning signs are not always observable in the environment, because they often only play out in the client's head. That is why a methodical risk management approach, such as ERM, is needed to identify and describe both observable and non-observable signs.

\section{The ERM and the Multivoiced Self}

Apart from identifying personalised early warning signs, ERM is useful because it offers a framework to systematically manage both internal and external dialogues with the client about the onset and proceedings of the process of deterioration. By means of the ERM, attention is given to interactions with and about client's perception and behaviours in a socalled 'multivoicedness' sense. This means that the ERM helps prisoners reflect on the dialogue between self and the voice of internal and external others as a means of managing their risk of violent behaviour. The prisoners voice and that of the differing professionals supporting them are captured and expressed when articulating their early warning signs of aggression and how to control these. Moreover, by means of ERM the 
prisoner is encouraged to engage in a process of self-reflection; the voices in himself are explored and articulated related to aggression. This will be explained by the following theory of the Self.

Within a person, the individualistic 'Self', the awareness of one own perception, is always infused with and responding to voices of others, referred to as 'inner Others'. The Self alters depending on whom the person interacts (Kohut, 1984; Aveling et al., 2015). For example, a client could articulate that he got angry because he thought that the staff disliked and were harassing him. His awareness of anger represents his Self, his I-position. However his conviction about staff represents his 'inner Others'. When evaluating a client's ERM-early warning signs, it is important to explore both what he experiences within himself (internal dialogue) but also the way he then relates to his environment (external dialogue), as we have seen in the General Aggression Model and Hiday model (see above). The relation between the Self and Inner Others is the carrier where aggression could begin with early warning signs. More on the 'Self' and the 'Self Theory' (Kohut, 1984) and how this has been used to analyse ERM interactions and the impact on clients aggressive behaviours can be found elsewhere (Fluttert et al., submitted 2020).

\section{Recognising Deteriorating Behaviours}

The ERM assessment tool is approached from the perspective of deteriorating behaviour, in line with the Hiday model. Special attention is paid to the social and interpersonal factors related to the individual behaving violently. From this perspective, the thoughts, feelings and behaviour of the patient can indicate the onset of aggression, and early recognition of these warning signs can help thwart such deterioration. The ERM hence draws further upon theory of social competence. Bartels (2001) developed a model of how patients in forensic care can react with violence when thoughts, feelings and behaviours associated with specific events interfere with their life skills and tasks. Stated simply, individual perceptions and reactions to events are influenced by personality characteristics, life experiences and interpersonal skills. So-called core beliefs (or personal convictions) seem to play a profound role in the shaping 
of reactions to events. Furthermore, the chain linking the perceptions of events and core beliefs to expressed behaviour can be referred to as a scenario. For patients with a personality disorder, a scenario can be grounded in certain core beliefs and related feelings of anger and thereby elicit accusations, threats or teasing. A profound insight into a patient's ability/competence to cope with stressful situations (e.g., due to delusions), and into the developmental process of aggression, is essential in the dynamic interactional understanding of violence (Bjørkly, 2006).

The central vision behind ERM is that disruptive behaviour, including aggression, develops gradually and that, especially, in the first phase of behavioural disruption, there are opportunities for intervening and stabilising the client's behaviour (see Fig. 11.1 Process of deterioration). Early warning signs of aggression can be defined as changes in individual, thoughts, perceptions, feelings and behaviours of the patient that fall along a spectrum ending at the crisis point that precipitates aggressive

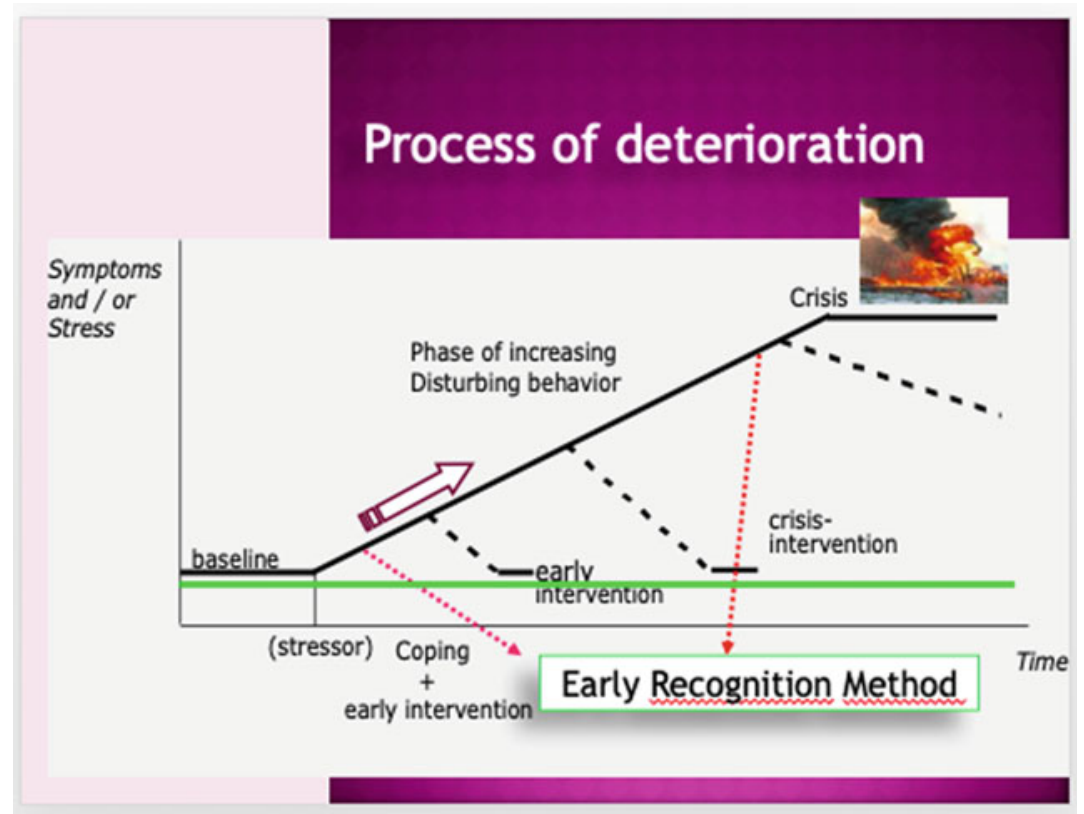

Fig. 11.1 Process of deterioration 
behaviour (Fluttert et al., 2008). In addition to the detection of early warning signs it is important to describe the context in which the early warning signs mainly occur. For example, the early warning signal of violence: 'irritation and anger' could occur especially when too many people surround the patient.

The baseline in the figure shows the behaviour as we encounter it in stable normal situations. The sloping line symbolises an increasing deterioration of behaviour. Normally we are able to restore ourselves to functioning on the baseline. However, when the stress and/or symptoms of a disorder increase, the behaviour can deteriorate further to a point where no adjustment/de-escalation is possible and a crisis is likely to occur. When applying ERM-plans, we focus on the functional area in the middle of the ascending line. In this area the behaviour is out of balance, but not completely deteriorated. This is the area where the first signs, the so-called early warning signs, occur. This is also the area in which there, pre-eminently, are opportunities for influencing behaviour and prevention.

Behavioural disruption usually does not arise from one moment to the next. Usually there are already a few days, or sometimes weeks in advance, when early warning signs are already beginning to announce instability. This may involve changes in thinking, feeling and /or behaviour. The early detection of such early warning signs makes it possible to intervene preventively by means of so-called early interventions. All data concerning early warning signs and early interventions are recorded in an ERM-plan.

In an ideal situation, prisoners and staff would collaborate in risk management and complete the ERM plan together. However, in reality this is not always structurally feasible due to lack of staffing and staff training. Nevertheless, preliminary pilots applying ERM in prisons in Norwegian and Dutch prisons show that when staff have the ERMknowledge they start more, and more meaningful interactions with prisoners who are showing aggression problems. 


\section{Models of ERM-Plans}

Working with ERM-plans has its origins in the treatment of patients with schizophrenia. Birchwood (2000) described how early warning signs in an ERM-plan could be described so that patients themselves learn to recognise these signs and thus prevent psychoses.

In the Netherlands there was increasing attention in the nineties for ERM-plans based on early warning signs. To date four models of ERMplans can be distinguished:

1. The Basic model. The early warning signs are listed on a checklist. The role of the patient is passive.

2. The Phase model. The different phases of a psychosis or behavioural disorder are described, as well as the corresponding actions for the patient.

3. The Comprehensive model. The early warning signs are described, but also the factors that influence their recognition. These are patientown factors and factors in the social network. The patient and his social network are actively involved in applying the ERM-plan.

4. The Dynamic model. A further development of the comprehensive model is that, in addition to the early warning signs and factors in the patient and the social network, the factor 'context' is also highlighted. These are descriptions of specific circumstances in which early warning signs could occur. For example, a patient may notice that his increasing desire for alcohol occurs mainly when he feels more lonely and when he has less contact with family. These contexts are usually related to offence-related factors and thus fit with the criminogenic needs detailed in the RNR model (Fluttert \& Eidhammer, 2018).

Based on the work of Birchwood, and on the comprehensive model and its associated protocol, Van Meijel et al. (2006) developed the 'Early Signs and Early Intervention Method' for general psychiatry. Fluttert et al. (2008) used this as the basis for the forensic variant and the dynamic model, the Early Recognition Method. ERM has not only been used and studied in mental health care since then, but also was studied 
in relation to how ERM risk management could be applied to prisoners and what this yields (Fluttert \& Eidhammer, 2018).

\section{Generations of Risk Assessment}

Thus far, we have described how violence and aggression could be understood in terms of knowledge structures, thinking styles and multiple factors in the context of the individual. We have also explored some of the theoretical underpinnings of the ERM. Next, we will bridge this knowledge to risk management strategies, and in particular, the ERM. Risk assessment in forensic psychiatry is an activity to assess and describe the extent and nature of offending. This is followed by risk management, the activity of enabling clients to manage and control disruptions in their behaviours. In the ideal scenario, risk assessment and risk management activities are carried out together.

Risk management strategies have undergone enormous development over the past decades. A distinction is made between three generations of risk management strategies:

1. The Unstructured Clinical Judgement: clinical judgement whereby the worker, from his perspective on the client, gives an opinion about the expected danger.

2. The Actuarial application: the application of risk assessment instruments whereby fixed items of behaviour are scored and the final score gives an indication of the expected risk.

3. Structured Professional Judgement (SPJ): whereby agreement is reached between two independent assessors. This is done in a structured manner on the expected recurrence of relapse of violence. The latter is developed on the basis of scored items from risk assessment instruments merged with clinical judgement (Douglas et al., 2013). The aim is to formulate the risk in the most accurate way explaining the client's personalised risk. This so-called 'risk formulation' is a description of possible scenarios of relapse (best case-, worst case-, relapseand twist scenarios) and risk interventions that help prevent relapse. A twist scenario is an unexpected change of the development of the risk, 
e.g. a client who was initially convicted for pedophilia could 'twist' towards more fantasies of sexual abuse with the possible outcome of killing children. The SPJ is the most dynamic and recent approach to risk management. The use of risk assessment instruments is linked in a structured way to risk management strategies. The application of ERM fits well with SPJ in that it determines the early warning signs in relation to risk scenarios. For example, if a client has described a risk scenario in which the use of alcohol is related to violent behaviour, then, while determining the early warning signs, the client will discuss which signs precede the search for or use of alcohol. The HCR-20V3 (see also Chapter 10 of this volume) is the world's most used risk assessment instrument fitting in the 3rd generation risk assessment (Douglas et al., 2013). This risk assessment instrument enables clinicians in a structured systematic way to assess and rate the most important items related to violence risk on the levels of low, moderate and high risk. A 7 step decision process is articulated within the HCR-20-V3, describing how the instrument should be applied in order to maximise the accuracy of the violence risk assessment.

The ERM is referred to within these 7 steps in the HCR-20V3 manual, referring to it as a promising method to apply the risk management part of the process. The value of ERM is seen as a follow up to the risk assessment conducted in the HCR-20-V3, and recognises the importance of the involvement of the client in assessing and formulating the risk (Ray \& Simpson, 2019). This makes it an instrument with potential to stimulate collaboration, interaction and multivoicedness as is very much stressed in the ERM protocol and training session.

\section{The Application of the Early Recognition Method [ERM]}

So far, ERM-plans have been described as has how ERM is ideally applied. But how is this applicable for prison staff and prisoners? To highlight this, we first consider the principles of ERM (henceforth, the terms supervisor and client are used). Then we can focus more specifically on ERM for prisoners. 
An important aspect of risk management strategies such as ERM is that the supervisor is aware of his or her own basic attitude towards clients. Clients in a closed institution who show resistance or hostility benefit from supervisors who do not immediately judge their behaviour but encourage the client to discuss what is bothering him or her (Meehan et al., 2006). Obviously, structure must be provided to inhibit, for example, scolding, shouting or threatening. At the same time, a client does not have to be motivated externally to be able to cooperate with ERM-plans. Internal motivation is also a form of motivation, e.g. that motivation can increase as a function of the client noticing that working with ERM-plans is not as complex or threatening as he initially thought.

The two most important factors in the process of working with ERM-plans are: (1) the client learns to accept that risk management conversations such as with ERM are not punitive or threatening, and (2) the client learns how to work with ERM-plans, which support him. The enduring benefit of ERM is about 'understanding', and that takes time. ERM-research (Fluttert et al., 2010a) shows that the discussion between client and staff regarding early warning signs is contributing to gain a better, and shared, understanding of the client's perceptions and behaviours. Even when they disagree about the occurrence of the early warning signs, pondering about 'rights' and 'wrongs' (of which there are none), these discussions enlighten client's behaviours in stability and less stable conditions.

The 'ERM- protocol' describes the strategy for guiding the client in the right way when drafting and implementing an ERM-plan. Such a protocol is necessary for supervisors in order to apply the method in a systematic manner, with the right steps and at the right time (Fluttert et al., 2016). The ERM protocol is based on sound scientific intervention research (Meijel et al., 2003; Fluttert et al., 2010b, 2013). Because the ERM focuses on the interaction in relation to early warning signs, this is pre-eminently a dialogue-based strategy. Several studies show that the interaction between care provider and client is the most important factor in influencing aggression during admission. The research into the application of the ERM also showed that weekly discussions between supervisors and clients had a meaningful contribution to reducing the number and severity of aggression incidents (Fluttert et al., 2010a). 


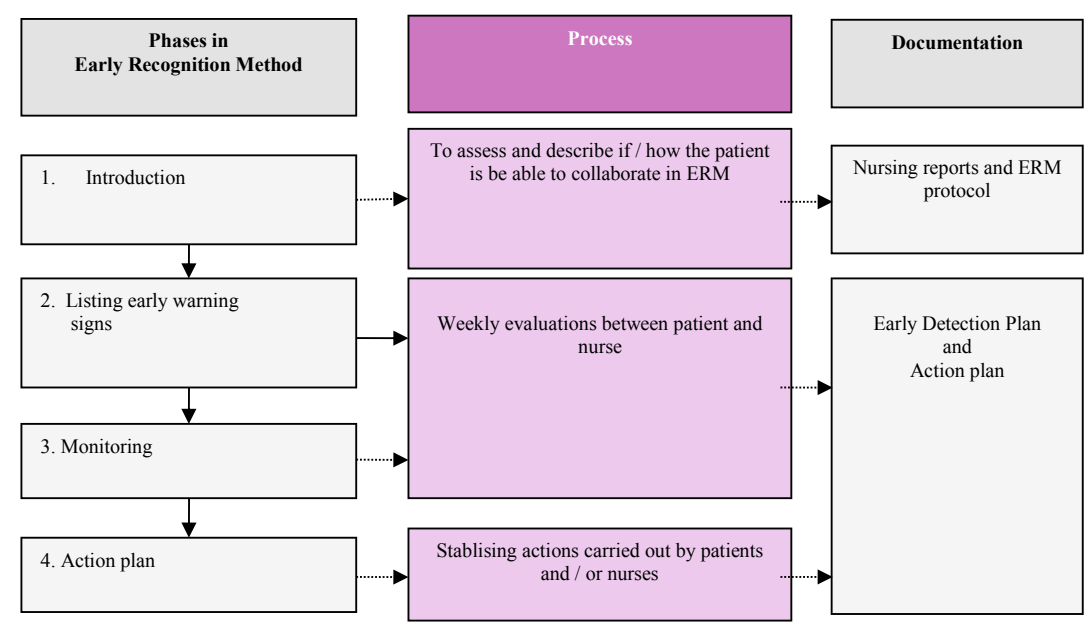

Fig. 11.2 ERM framework

The work protocol (see Fig. 11.2 ERM framework) associated with the ERM consists of four phases, i.e. the steps in which the method is applied: (1) introduction phase, (2) identification phase, (3) monitoring phase and (4) action phase.

\section{Phases of the Protocol}

Introduction/preparation phase

The professional explains to the client/prisoner the purpose of the ERM, the ERM-plan and what is expected of him. At the same time, it is assessed at this stage whether and how the client will be able to work with the ERM-plan. The strategy, the way in which ERM is applied, is then decided. If necessary, an ERM-plan can be drawn up without the client's cooperation.

Identification phase

In this phase, early warning signs are listed and described in the ERMplan. Each early warning sign is described at three levels of severity, (1. stable, 2. disrupted and 3. more disrupted). The means by which the client can learn to recognise their own relevant early warning signal and 
the levels of severity of these are then articulated. A major obstacle to this process is that there are hardly any instruments available to support clients and supervisors to identify the relevant early warning signs of aggression in a structured manner. The FESAI (Forensic Early Signs or Aggression Inventory) was hence developed as a tool to help clients identify their own early warning signs. In the construction of the FESAI, 167 ERM-plans and 3768 descriptions of early warning signs were studied and then categorised. The validity and 'inter-rater reliability' were tested and assessed as sufficiently adequate and reliable (Fluttert et al., 2011, 2013). The FESAI is a list of 44 items of possible early warning signs divided over 14 main categories, namely:

1. Change in daily activities.

2. Social isolation/decreased social contact.

3. Change of self-management.

4. Physical changes.

5. Changed substance needs (alcohol, drugs, medication).

6. Cognitive changes.

7. Dejection and anxiety.

8. Tension, agitation, anger.

9. Non Violent anti-social behaviour.

10. Disinhibition and impulsivity.

11. More (extreme) sexual fantasies /needs /behaviours.

12. Criminal behaviour.

13. Irrational ideas/perceptions.

14. Very specific changes of behaviours.

Specific warning signs are described within these categories. Monitoring phase

The monitoring phase involves scanning of the prisoners' behaviour with the aim of recognising the occurrence of early warning signs. The dialogue between the client and the supervisor about the occurrence of early warning signs takes place here. In this phase, discussions often take place between client and supervisor, often from a disagreement about the assessment of the occurrence of the early warning signal. The trained and professional supervisor remains neutral, and tries to explore together 
with the client how he interprets his behaviour and creates a dialogue about the differences of perception of the same behaviour.

Action phase.

Finally, if possible, an action plan is drawn up together with the client that is part of the ERM-plan. It describes which actions can contribute to stabilisation of behaviour.

The competences required to apply ERM can be trained in a customised ERM-training programme developed for nurses working in (forensic) psychiatry or prison officers in prisons. In these training sessions the staff learns the basic theory behind aggression and violence (as explained earlier) and how to identify and manage early warning signs by means of the ERM protocol.

\section{The ERM as a Tool for Client Involvement and Shared Decision-Making in Risk Management}

In recent years there has been more recognition of the client's voice and involvement in treatment planning (Gudde et al., 2015). This is a development that makes clients more assertive with respect to privacy and the right of access to, for example, treatment plans. In addition, current internet use contributes to better access for clients in terms of knowledge about, for example, disorders and treatment options. Social media platforms like Google and Wikipedia are examples of such sources that are often consulted. But also from an ethical perspective, and through a more effective use of care, care providers are more aware of the active role of clients in their guidance and treatment. This also applies to prisoners who, similarly, are aware of privacy rights and the possibilities of media platforms.

'Shared decision-making' [SDM] is an approach based on the principle that there are two experts in care and treatment: (1) the client and (2) the care provider (Legare et al., 2011). We will first explain the SDM model (thus use the term 'care provider'), and next, reflect how this could be valuable when applying ERM in prisons by prison-officers who are involved in taking care of the security in prisons. 
The care providers have expertise in the process of diagnosis, risk assessment and risk management. Clients are the experts when it comes to experiences with their problems, i.e. what helps them and what gives meaning to the quality of their lives. Ideally, care providers and clients agree on the nature and purpose of the treatment and risk management. Through an active participation of the client in the treatment programme, the chances are increased that choices are made that suit the client better, to which he or she can connect better, so that the effectiveness increases. There is now enough research in mental health care that show that clients benefit from a process of SDM (Patel et al., 2008). For example, in a systematic review Patel et al. found that in an RCT-study among psychiatric patients, SDM resulted in a trend towards reduced rehospitalisation rates and improvement of self-efficacy of symptom management of mental illness. Also in forensic services, the collaboration between the forensic worker and the client is intended to eventually teach the client to independently control his risk (Kroner, 2012). However, in contrast to the principles of SDM, Eidhammer et al., 2014 showed that patient involvement is scarce in evidence-based risk management strategies. In this review, only the following three risk management strategies were found where the client had an active role in its application: (1) ProLad, a step-wise forward systematic rehabilitation programme (2) ERM, and (3) Anger Management programmes. The ERM, in collaboration with forensic patients, identifies early warning signs whereby the patient gets a better understanding of his deteriorating behaviours.

SDM is a relevant in prison services also, the basic assumption being again that the prisoner is an expert of his own needs or symptoms. In prisons often the prison officers 'know' by intuition when and how prisoners become aggressive. When this 'tacit knowledge' is articulated by describing early warning signs, then prisoners' behaviours could be more accurately described and monitored. This intuition should be harnessed and 'translated' into observable behaviours and recognised in a timely fashion. The ERM can be used as a SDM strategy that 'opens doors' and starts a systematic collaboration between prison staff and prisoners exploring jointly how stability could potentially deteriorate towards aggression. Training for all prison staff is advised before 
applying risk management strategies such as ERM. In a pilot study in the Netherlands and Norway ERM was taught to prison staff, and in some cases, they were also taught how to collaborate with prisoners in these endeavours. Preliminary results show that prison officers value working with ERM positively because their 'gut feelings' about the prisoners are made concrete and thereby they were better able to cooperate effectively with the prisoner. The ultimate goal would be to train prison officers systematically to gain knowledge in practice of how to apply risk management, such as ERM, resulting in a better management and prevention of aggression in those institutions.

\section{Transfer of ERM-Knowledge Between Forensic and Prison Services}

In Norway and the Netherlands there has been interest in the use of ERM among prisoners for several years. The reasons for this transfer of knowledge from the Forensic Psychiatry to Prison context is a need to reduce the occurrence of crisis situations in the prison as well as a tool for professionalisation of prison staff who have an increasingly need for higher competence in risk management as a methodical approach.

Initially the Early Signs and Early Intervention Method was developed for patients with Schizophrenia in general psychiatry (Birchwood, 2000; van Meijel et al., 2006). Elaborating on this concept Fluttert et al. (2008) developed the Forensic ERM version, applied this and studied this renewed ERM intensively. The prison services then showed interest and a process of studying, revising and transfer started in order to develop an ERM Prison version. The main steps are described below. A pilot was done in order to gain knowledge whether the multivoicedness resulted in a ERM version which fits to prison services. The final ERM version appeared to contribute to the collaboration between prison staff but also between prison staff and prisoners. Although the prison is often seen as a non-specialist service, the ERM enabled prison staff to enlarge their knowledge about aggression and how to manage it. Prison staff became prison specialists in ERM. The ERM also acknowledges that all contributing to its completion are experts in their own area whether they 
be forensic mental health nurses, researchers, prison staff and as such have a contribution to make to the assessment and management of risk.

In Norway, two pilot projects were launched between 2014 and 2016 in prisons in Hustad and Oslo Bredtveit Prison (the latter being a women's prison for long-term prisoners). In these projects, the ERM protocol, as developed for forensic psychiatric clinics, was revised so that an application for the context of penitentiary work became available. This revision process was undertaken as follows:

1. The first step was to formulate with the prison leaders a first draft of a project plan in which the aims, available project resources and research were agreed. At the Oslo-SIFER-Expertise Centre, Norway and at Molde University College, Norway, ERM-project groups were established that worked on revising the 'Forensic ERM-protocol' into a 'Prison ERM protocol' and assisting the prison services in implementing and applying ERM. In each prison a 'key-person' was assigned to be in contact between the prison staff and the expert group.

2. Regional ethical committees' permissions were obtained to run the study.

3. ERM training seminars for the prisons staff served as 'kick-off' meetings; prison staff got familiar with the basis assumption of ERM and how it could be valuable for their work with prisoners.

4. In collaboration between the ERM-project group and the prison, staff discussed revisions to the ERM protocol for prisoners. Apart from the local context, the risk assessment conducted with the model combined the criteria of the RNR model with the conceptual assumptions of ERM (see above) This lead to the first draft of the 'ERM-protocol-Prison version NO'.

5. The first ERM prison protocol was in both prisons applied during 1 year. During this year there were interview-meetings between project group members and prison staff exploring their perceptions of the potential of using the ERM. These discussions lead to adjustments in the protocol mostly concerning the use of terminology and more emphasis being placed on observing behaviours by means of the FESAI. 
One year after the start of the pilots a concluding meeting was held between the project group and prison delegates. The project was evaluated and followup actions formulated how ERM to embed in prison practice. Details of these evaluations are reported elsewhere (Eidhammer et al., 2013) but overall interviews with prison staff showed they had found the use ERM a beneficial learning experience. This had been the first project they had experienced in which a particular risk management strategy (ERM) had been systematically discussed with them and through which knowledge and experiences between the forensic and prison fields had been exchanged.

In applying the ERM, and comparing this to the application of the ERM in the FPC, the Prison version of ERM focused more on identifying and observing early warning signs and less on the therapeutic relationship between supervisor and prisoner. Despite the staff being encouraged to discuss early warning signs with prisoners, observations and recordings of signs were more central. The ERM had provided them with a 'tool' to get a grip of risk signs in a structured way. Their daily 'usual' observations and impressions of the offender could now be articulated as early warning signs. The Prison version of the ERM working protocol, transformed into a kind of 'light version', meaning that the therapeutic interactions were re-formulated into directives and instructions and thereby better fitting to the context of prison staff.

Risk management skills are pivotal to the application of risk management strategies such as ERM. Prison staff were well prepared in this regard as they are already trained in monitoring and promoting safety in the organisation and the secured environment. They also expect today to have a greater contribution to make to the rehabilitation of prisoners (Osment 2018). This requires interaction and observation skills, not only to motivate the prisoner to cooperate, but also to be able to observe and manage possible behavioural disruptions in a timely manner. When using ERM in forensic psychiatry, the emphasis is on the interaction between therapist and patient. When applying it in prison institutions, the emphasis is more on making explicit intuitive actions on the basis of experience with prisoners, due to a lack of systematic risk management strategies. If possible, this will be discussed with the prisoner, but such risk management discussions between prison staff and prisoners are not 
yet customary. The experience so far is that ERM contributes to prison staff being able to systematically make their intuitive observations explicit by describing early warning signs and registering them in the ERM-plan.

Of similar importance is the observation that ERM contributes to the interaction between prison staff and prisoner. When prison staff are able to build up interactions with prisoners in a structured methodical way, this contributes to better cooperation and also a more positive image of prison staff among prisoners (Crewe et al., 2011). Thus, prison staff are not solely providing safety and security, but can also be instrumental in prisoners developing more sustainable and stable behaviours. Ultimately, enabling the prisoners' rehabilitation back into society. Despite the difference between prison staff and mental health workers, with prison staff having to cover also social work issues, there are also similarities. Both disciplines are at the front line in their institutions. They are both in the position to observe, intervene and collaborate with the clients in case of deteriorating behaviours. For patients and prisoners, the 'frontline' workers are those who care for them 24/7 and thereby have the best opportunity to start systematic risk formulation interactions based on daily observations and experiences. This makes prison officers ideal resources when mental health professionals seek input for identifying and managing ERM-early warning signs.

\section{Preliminary Findings on the Application of ERM Among Prisoners}

The first results of the Norwegian ERM-pilot studies are encouraging. After a very short instruction session (lasting a few hours) it appeared that the prison staff could use the ERM-plan to describe early warning signs, including describing, at three severity levels, how the signal can be recognised. Furthermore, the FESAI appeared to provide good insight and guidance to determine the person-specific early warning signs per prisoner. In addition to the prison staff describing the signs in their own words, the FESAI immediately added a code corresponding to the category and the item in question in the FESAI. This provides opportunities for more systematic investigations into the ranking and classification of 
early warning signs in prisoners. It also appears that the format/layout of the ERM-plan provides sufficient guidance for adequately scoring the occurrence of warning signs over time, i.e. the so-called monitoring of behaviour.

A first rank-order analysis showed that the most common early warning signs had a strikingly similar ranking compared to forensic psychiatry (Fluttert, 2015). In other words, like in forensic psychiatry, the most prevalent early warning signs registered with prisoners were: (1) Tense, agitation and anger, (2) withdrawal from contact and fewer contacts and (3) change in daily activities. In the pilot study in Norway, the prison staff (Eidhammer et al., 2013) indicated that since the implementation of ERM they had gained much more insight into the significance and meaning of prisoners withdrawing. Previously, this behaviour was often seen as 'he is quiet, more in the background'. Now, this is acknowledged as a possible early warning sign in the sense that 'he feels less at ease and tolerates others less well'. The 'withdrawal behaviour' now has a different meaning within the framework of ERM and practice has shifted towards making contact with this prisoner early on in order to determine what is on his mind. Undoubtedly, this is a good starting point to engage in risk management.

In an evaluation in 2017 of the application of ERM observations in the Norwegian women's prison it emerged that staff had started to discuss the ERM-plan with the prisoners, as a natural next step. Although there is no question of a therapeutic interaction, the practical application of discussing the ERM-plan with the prisoner leads to better cooperation between prisoners and staff. An example of this is a prisoner who, for a long time, avoided contact with both prison staff within the institution and with the contact person in the rehabilitation programmes. This prisoner said that through the ERM talks, she gradually began to see that this form of risk management could support her in an existence 'outside' the institution. This insight led to her taking the initiative to discuss her ERM-plan with the contact person 'outside'. However, on the basis of such examples, we do not yet know anything about the effect of ERM on improving the stability of prisoners in the longer term. But, insofar the use of risk management strategies is meaningful for prisoners, and the interaction therein is important (Crewe et al., 2011), it is not 
unlikely that the ERM is a hope-inducing approach that can facilitate social rehabilitation.

\section{Summary and Conclusions}

Working in closed institutions requires a lot of commitment and professionalism from employees when dealing with stress. The likelihood of burnout is present in both prisons and institutions of forensic psychiatry. Actors in both environments have experiences of aggression or feeling 'something is about to happen' with respect to violence.

Aggression and violence (risk) appear regardless of where the person (patient or prisoner) is admitted. The application of risk management strategies contributes to the timely recognition of behavioural deterioration and the ability to manage them. The ERM is a risk management strategy that enables staff to recognise and manage early warning signs of deterioration in a timely manner. In forensic psychiatry, there are ample experiences of ERM in relation to its clinical relevance (Fluttert et al., 2010b, 2013) efficiency in terms of better incident management (Ray \& Simpson, 2019).

In this chapter, we suggest the ERM has innovative potential to be transferred as a model into prison environment. Pilots of the ERM in Norway have shown that the ERM contributes to better understanding and control among prison staff of behavioural deterioration in prisoners. Prison staff have learned how to use a structured risk management interaction through ERM. Central to this risk management is the awareness of the occurrence of early warning signs and then the possible application of stabilising early interventions as a response to the onset of behavioural disturbance. We believe that this approach may potentially have utility to prisons across Europe, and might, ultimately, contribute to both safety within the prisons and the rehabilitation of prisoners.

The core element of the ERM-methodology is to identify and adapt new behavioural strategies through direct involvement of the service user. Thus, "multivoiced" collaboration is extensively explored and highlighted in this chapter. The prisoner explores the Self in their hetero 
and auto dialogues with multiple voices in their environment (Avedeling et al., 2015). Some of these dialogues may trigger violent episodes and need to be addressed before these take hold. The ERM is a means of shared decision-making that can involve specialists (mental health professionals), non-specialists (e.g. prison officers) and the prisoner themselves. It acts as a boundary object through which these stakeholders can cooperate in the interest and safety of all involved.

The ERM is a new model of risk management being applied to the prison context and although preliminary pilots are favourable, more intensive research is required to explore its effectiveness in the prison environment. Doing so, in this new context, will contribute to an even more comprehensive conceptualisation of aggression and violence, and the management and prevention of these. The main challenges to these future endeavours may be a 'paradigm issue' however, i.e. how are prison staff able to put aside their focus on safety and security and switch to a more interactional or caring approach to working with inmates. Training will be required to prepare them for working in a coordinated and systematic manner that enables them to assess and manage instruments, such as the ERM, innovations borrowed from the mental health field.

\section{References}

Andersen, D. R., Andersen, L. P., Gadegaard, C. A., Høgh, A., Prieur, A., \& Lund, T. (2017). Burnout among Danish prison personnel: A question of quantitative and emotional demands. Scandinavian Journal of. Public Health., 45(8), 824-830.

Andrews, D. A. (2012). The risk-need-responsivity (RNR) model of correctional assessment and treatment. In J. A. Dvoskin, J. L. Skeem, R. W. Novaco, \& K. S. Douglas (Eds.), Using social science to reduce violent offending (pp. 127-156). New York, NY: Oxford University Press.

Aveling, E. L., Gillespie, A., \& Cornish, F. (2015) A qualitative method for analysing multivoicedness. Qualitative Research, 15(6), 670-687.

Bartels, A. A. J. (2001). The model of social competence in the treatment of forensic youth psychiatry, an in-depth study. In Antisocial behaviour 
of youngsters. Determinants and interventions (ch. 6, pp. 95-124). (Het sociale-competentiemodel in forensische jeugdpsychiatrische behandeling; verdieping en verbreding. In: Antisociaal gedrag bij jeugdigen. Determinanten en interventies). Lisse: Swet \& Zeitlinger.

Bezerra, C., \& De Mahalhães. (2016). Psychological distress and work stress in correctional officers: A literature review. Ciência \& Saúde Coletiva, 21(7). https://doi.org/10.1590/1413-7.00502016.

Birchwood, M. (2000). The critical period for early intervention. In M. Birchwood, D. Fowler, \& C. Jackson (Eds.), Early intervention in psychosis. Chichester: Wiley.

Bjørkly, S. (2006). Empirical evidence of a relationship between insight and risk of violence in the mentally ill; A review of the literature. Aggression and Violent Behavior, 11, 414-423.

Bjørkly, S., Wærstad, J. M., Selmer, L. E., Wærp, J., Bjørnstad, M., Leinslie, J. V., et al. (2019). Violence after discharge from forensic units in the safe pilot study: A prospective study with matched pair design. Psychology Research and Behaviour Management., 12, 755-766.

Bulten, E., Nijman, H., \& Van der Staak, C. (2009). Measuring criminal thinking styles; The construct validity and utility of the PICTS in a Dutch prison sample. Legal and Criminological Psychology, 14, 35-49.

Campbell, J., Sellen, J. L., \& Macmurran, M. (2010). Personal Aspirations and Concerns Inventory for Offenders: Developements in the measurement of offenders' motivation. Criminal Behaviour and Mental Health, 20, 144-157.

Carlsson, G., Dahlberg, K., Ekebergh, M., \& Dahlberg, H. (2006). Patients longing for authentic personal care: A phenomenological study of violent encounters in psychiatric settings. Issues in Mental Health Nursing, 27, 287305.

Crewe, B., Liebling, A., \& Hulley, S. (2011). Staff Culture, use of authority and prisoner quality of life in pubic and private sector prisons. Australian \& New Zealand Journal of Criminology, 44(1), 94-115.

Douglas, K. S., Hart, S., Webster, Ch., Belfrage, H., Guy, L., \& Wilson, C. (2013). Historical-Clinical_Risk Management-20, Version 3 (HCR-20V3): Development and overview. International Journal of Forensic Mental Health, 13, 93-108.

Eidhammer, G., Fluttert, F., \& Bjørkly, S. (2014). User involvement in structured violence risk management within forensic mental health facilities-A systematic literature review. Journal of Clinical Nursing, 23, 2716-2724. 
Eidhammer, G., Fluttert, F., Knutzen, M., \& Bjørkly, S. (2013). Report: Early recognition method ERM, Pilotfase 2-2009-2013. Kompetansesenter for Sikkerhets-fengels og Rettspsykiatri for Helseregion Sør-Øst.

European Commission. (2013). Guide to social innovation. Brussels: EU commission. Available at https://ec.europa.eu/eip/ageing/library/guide-soc ial-innovation_en. Accessed 1 August 2020.

Finney, C., Stergiopoulos, E., Hensel, J., Bonato, S., \& Dewa, C. (2013). Organizational stressors associated with job stress and burnout in correctional officers: A systematic review. BMC Public Health, 13, 82.

Fluttert, F., Van Meijel, B., Bjørkly, S., Van Leeuwen, M., Nijman, H., \& Grypdonck, M. (2013). Early signs of inpatient aggression in forensic psychiatry: An empirical investigation with the Forensic Early Signs of Aggression Inventory [FESAI]. Journal of Clinical Nursing, 22(11-12), 1550-1558.

Fluttert, F., Van Meijel, B., Van Leeuwen, M., Bjørkly, S., Nijman, H., Grypdonck, M. (2011). The development of the Forensic Early warning Signs of Aggression lnventory [FESAI]: Preliminary findings. Towards a better management of inpatient aggression. Archives of Psychiatric Nursing, 25(2), 129-137.

Fluttert, F., Van Meijel, B., Nijman, H., Bjørkly, S., \& Grypdonck, M. (2010a). Detached concern of Forensic Mental Health Nurses to patients with regard to the Early Recognition Method. Archives of Psychiatric Nursing, 24(4), 266-274.

Fluttert, F., Van Meijel, B., Nijman, H., Bjørkly, S., \& Grypdonck, M. (2010b). Effect of The Early Recognition Method on the number of aggressive incidents and episodes of seclusions in forensic care. Journal Clinical Nursing, 19, 1529-1537.

Fluttert, F., Van Meijel, B., Nijman, H., Webster, C., \& Grypdonck, M. (2008). Risk management by early recognition of warning signs in forensic psychiatric patients. Archives of Psychiatric Nursing, 22(4), 208-216.

Fluttert, F. A. J. (2015). Early Recognition Method in prison services. Presentation at the Conference of the International Association Forensic Mental Health Services, Manchester, UK.

Fluttert, F. A. J., Bjørkly, S., \& Dale, K. Y. (2020). The impact of a discarded diagnosis and focus on early warning signs of aggression on relations between user and municipal service providers-A narrative of a complex case. Current Psychology.https://doi.org/10.1007/s12144-020-01055-x. 
Fluttert, F. A. J., \& Eidhammer, G. (2018). ERM-Vroegsignalering opent de deur naar risicomanagement observaties en de dialoog met gedetineerden. Proces, 97-1, 25-39.

Gadon, L., Johnstone, L., \& Cooke, D. (2006). Situational variables and institutional violence: A systematic review of the literature. Clinical Psychological. Review., 26(5), 515-534.

Gudde, C. B., Oslø, T. M., Whittington, R., \& Vatne, S. (2015). Services users' experiences and views of aggressive situations in mental health care: A systematic review and thematic synthesis of qualitative studies. Journal of Multidisciplinary Healthcare, 8, 449-462.

Hiday, V. A. (1997). Understanding the connection between mental illness and violence. International Journal of Law and Psychiatry, 20, 399-417.

Hiday, V. A. (2006). Putting community risk in perspective: A look at correlations, causes and controls. International Journal of Law Psychiatry, 29(4), 316-331.

Kohut, H. (1984). How does analysis cure? Chicago: University of Chicago Press.

Kroner, D. G. (2012). Service user involvement in risk assessment and management: The transition inventory. Criminal Behavior and Mental Health, 22, 136-147.

Legare, F., Stacey, D., Pouliot, S., Gauvin, F. P., Desroches, S. et al. (2011). Interprofessionalism and shared decisionmaking in primary care: A stepwise approach towards a new model. Journal of Interprofessional Care, 25, 18-25. Looman, J., \& Abracen, J. (2013) The risk need responsivity model of offender rehabilitation: Is there really a need for a paradigm shift? International Journal of Behavioural Consultation and Therapy, 8(3-4), 30-36.

van Leeuwen, M. E., \& Harte, J. (2016). Violence against mental health care professionals: Prevalence, nature and consequence. The Journal of Forensic Psychiatry and Psychology., 28, 581-598.

Meehan, T., McIntosh, W., \& Bergen, H. (2006). Aggressive behaviour in the high secure forensic setting: The perceptions of patients. Journal of Psychiatric and Mental Health Nursing, 13, 19-25.

van Meijel, B., Kruitwagen, C., van der Gaag, M., Kahn, R., \& Grypdonck, M. (2006). An intervention study to prevent relapse in patients With Schizophrenia. Journal Nursing Scholarship, 38(1), 42-49.

van Meijel, B., van der Gaag, M., Kahn, R. S., \& Grypdonck, M. (2003). Relapse prevention in patients with schizophrenia. Archives of Psychiatric Nursing, 17, 117-25. 
Newbill, W. A., Marth, D., Coleman, J. C., Menditto, A. A., Carson, S. J., \& Beck, N. C. (2010). Direct observational coding of staff who are the victims of assault. Psychological Services, 7(3), 177-189.

Nijman, H., Allertz, W. F. F., Merckelbach, H. L. G. J., Campo, J. L. M. G., \& Ravelli, D. P. (1997). Aggressive behaviour on an acute psychiatric admission ward. European Journal of Psychiatry, 11(2), 106-114.

Osment. L. (2018). The complexity of rehabilitation in open and closed prison settings. Master Thesis, Lund University, Sociology of Law Department.

Patel, S. R., Bakken, S., \& Ruland, C. (2008). Recent advances in shared decision making for mental health. Current Opinion Psychiatry, 21, 606-612. Ray, I., \& Simpson, A. (2019). Shared risk formulation in forensic psychiatry. Journal American Academic Psychiatry Law, 47(1), 22-28.

Taxman, F., A. Pattavina, M. Caudy \& Joseph Durso (2013). The empirical basis for the RNR model and updated RNR conceptual framework. In F. S. Taxman \& A. Pattavina (Eds.), Simulation Strategies to Reduce Recidivism. New York: Springer Publishers.

United Nations Office of Drugs and Crime. (2016). Handbook on the crime of high risk prisoners. New York: United Nations Publications.

Ward, T. (2002). The management of risk and the design of good lives. Australian Psychologist, 37, 172-179.

Open Access This chapter is licensed under the terms of the Creative Commons Attribution 4.0 International License (http://creativecommons.org/ licenses/by/4.0/), which permits use, sharing, adaptation, distribution and reproduction in any medium or format, as long as you give appropriate credit to the original author(s) and the source, provide a link to the Creative Commons license and indicate if changes were made.

The images or other third party material in this chapter are included in the chapter's Creative Commons license, unless indicated otherwise in a credit line to the material. If material is not included in the chapter's Creative Commons license and your intended use is not permitted by statutory regulation or exceeds the permitted use, you will need to obtain permission directly from the copyright holder.

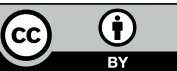

